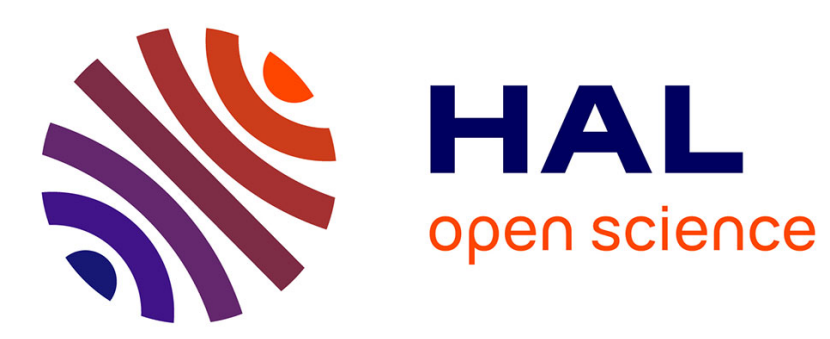

\title{
Convexity Preserving Contraction of Digital Sets
}

\author{
Lama Tarsissi, David Coeurjolly, Yukiko Kenmochi, Pascal Romon
}

\section{To cite this version:}

Lama Tarsissi, David Coeurjolly, Yukiko Kenmochi, Pascal Romon. Convexity Preserving Contraction of Digital Sets. The 5th Asian Conference on Pattern Recognition (ACPR 2019), Nov 2019, Auckland, New Zealand. pp.611-624, 10.1007/978-3-030-41299-9_48. hal-02315084v2

\section{HAL Id: hal-02315084 \\ https://hal.science/hal-02315084v2}

Submitted on 10 Mar 2020

HAL is a multi-disciplinary open access archive for the deposit and dissemination of scientific research documents, whether they are published or not. The documents may come from teaching and research institutions in France or abroad, or from public or private research centers.
L'archive ouverte pluridisciplinaire HAL, est destinée au dépôt et à la diffusion de documents scientifiques de niveau recherche, publiés ou non, émanant des établissements d'enseignement et de recherche français ou étrangers, des laboratoires publics ou privés. 


\title{
Convexity Preserving Contraction of Digital Sets ${ }^{\star}$
}

\author{
Lama Tarsissi ${ }^{1,2}$, David Coeurjolly ${ }^{3}$, Yukiko Kenmochi ${ }^{1}$, and Pascal Romon ${ }^{2}$ \\ 1 LIGM, Université Gustave Eiffel, CNRS, ESIEE Paris, Marne-la-vallée, France \\ 2 LAMA, Université Gustave Eiffel, CNRS, Marne-la-vallée, France \\ 3 Université de Lyon, CNRS, LIRIS, Lyon, France
}

\begin{abstract}
Convexity is one of the useful geometric properties of digital sets in digital image processing. There are various applications which require deforming digital convex sets while preserving their convexity. In this article, we consider the contraction of such digital sets by removing digital points one by one. For this aim, we use some tools of combinatorics on words to detect a set of removable points and to define such convexity-preserving contraction of a digital set as an operation of rewriting its boundary word. In order to chose one of removable points for each contraction step, we present three geometrical strategies, which are related to vertex angle and area changes. We also show experimental results of applying the methods to repair some non-convex digital sets, which are obtained by rotations of convex digital sets.
\end{abstract}

Keywords: Digital convexity $\cdot$ Digital set contraction $\cdot$ Christoffel words $\cdot$ Lyndon words

\section{Introduction}

Convexity is one of the useful geometric properties of digital sets in digital image processing. There are various applications which require deforming digital convex sets while preserving their convexity. In this article, we consider the contraction of such digital sets by removing digital points one by one. For this aim, we use some tools of combinatorics on words to detect a set of removable points and to define such convexity-preserving contraction of a digital set as an operation of re-writing its boundary word.

A relation between combinatorics on words [16] and digital geometry [15] has afforded many advantages to both areas and has led to interesting results. Indeed, such a connection realizes through the so-called Freeman coding, introduced by Freeman in 1961 [13], that allows to uniquely determine a 4- or 8-connected finite set of points in the discrete plane by means of its boundary word, i.e. a word over the alphabet of cardinal four $A=\{0,1,2,3\}$. This code is the bridge between these two worlds. In this article, we deal with one of the main notions of convexity for polyominos presenting in literature: digital convexity. During the

\footnotetext{
* This work was partly funded by the French National Research Agency, grant agreements ANR-10-LABX-58 (Labex Bézout) and ANR-15-CE40-0006 (CoMeDiC).
} 
last decades, many definitions were given; it started with Minsky and Papert in 1969 [18] and then Hubler (further notions and related studies can be found in $[4,5,9,10])$. A subset $C$ in a digital image is convex if the straight line segment joining any two pixels $P$ and $Q$ of $C$ lies entirely in $C$. We also present another notion of digital convexity with a help of the convex hull of $C$. In this article, we rely on a recent result by Berleck and al. [3], who have defined a set to be digitally convex if and only if the Lyndon factorization of its boundary word is made of Christoffel words. Based on this definition, we show how we can deflate a digitally convex set by choosing correct digital points to remove and preserving the convexity at the same time. We can find some previous studies on inflating digitally convex sets [11]. One of the main contributions of this article is to give three different strategies that allow us to determine an order to follow in removing digital points.

This article is decomposed into four parts. We first introduce digital convexity and give some related notions about two families of words. Then, we show the link between digital convexity and combinatorics on words. In the second part, we do modifications on the border of a digital convex set, and show all the perturbations obtained after removing a digital point. The third part is for presenting three different techniques in choosing a certain order for deflating digital convex sets. The last part is dedicated for showing experimental results, where we apply our methods to a rotated digital convex set, which is not convex anymore, in order to repair its convexity by making the convex hull and deflating it by removing some digital points.

\section{Digital convexity and combinatorics on words}

In this section, we introduce the basic notions needed in the following sections. Precisely, we recall basic notions in combinatorics on words. Then we define digital convexity and two particular families in combinatorics on words, that are Christoffel and Lyndon words [1,16].

\subsection{Basic notions of Combinatorics}

An alphabet $A$ is a finite set of symbols such that its elements are called letters. A word $w$ over an alphabet $A$ is a finite sequence of letters over $A$. In another way, a word $w$ is obtained by concatenating letters of $A$, we write $w \in A^{*}$, where $A^{*}$ represents the set of all the words formed by $A$, with the empty word $\epsilon$ being the identity element for the concatenation. We denote $w^{n} \in A^{*}$ the concatenation of the word $w, n$ times, such that: $w^{n}=w \cdot w \cdot w \cdots w$ where $w$ is repeated $n$ times with the convention of $w^{0}=\epsilon$. A word $w$ is said primitive if it is not the power of a nonempty word.

Let $w \in A^{*}$. The length of $w$ is the number of letters of $w$ denoted by $|w|$. Note that $|\epsilon|=0$. For all $\ell \in A,|w|_{\ell}$ denotes the number of occurrences of the letter $\ell$ in the word $w$, so that: $|w|=\sum_{\ell \in A}|w|_{\ell}$. 
Two words $w$ and $w^{\prime}$ are conjugate of order $k$, with $k \neq 0$, denoted by $w \equiv_{k} w^{\prime}$, if and only if there exist $u, v \in A^{*}$ such that $|u|=k, w=u . v$ and $w^{\prime}=v \cdot u$. When the exact value of $k$ is not relevant, we simply write $w \equiv w^{\prime}$. We can set several order relations over $A^{*}$. Here, we choose the total lexicographic order, or simply the dictionary order that is a total order and denoted by $<$.

Definition 1. Let $w_{1}, w_{2} \in A^{*}$, we say that:

$w_{1}<w_{2}$ if $\left\{\begin{array}{l}w_{2}=w_{1} \cdot w^{\prime}, \text { where } w^{\prime} \in A^{*} ; \text { or } \\ w_{1}=u . a . v_{1} \text { and } w_{2}=u . b . v_{2} \text { where } u, v_{1}, v_{2} \in A^{*} \text { and } a<b \in A .\end{array}\right.$

\subsection{Polyomino and boundary words}

Let us consider the lattice $\mathbb{Z}^{2}$, which is the set of all the vectors having integer components. The canonical basis of the Euclidean vector space $\mathbb{R}^{2}$ is $\left\{e_{1}, e_{2}\right\}$. We call by a finite discrete set any finite subset of $\mathbb{Z}^{2}$. We define a path in $\mathbb{Z}^{2}$ from point $X$ to point $Y$ a sequence of points $\left(p_{i}\right)_{1 \leq i \leq n}$ with $p_{i} \in \mathbb{Z}^{2}$ where $p_{1}=X$ and $p_{n}=Y$. A path in $\mathbb{Z}^{2}$ is 4 -connected if for any $1 \leq i \leq n-1$, $p_{i+1}-p_{i} \in\left\{ \pm e_{1}, \pm e_{2}\right\}$. A polyomino $P$ is a simply 4-connected finite set of digital points, without hole, that can be visualized as set of a unit squares. The Freeman chain code allows us to represent any path over $\mathbb{Z}^{2}$ as a word over an alphabet of four letters $A=\{0,1,2,3\}[13]$ such that:

- 0 denotes a right horizontal step;

- 2 denotes a left horizontal step;

- 1 denotes an upside vertical step;

- 3 denotes a downward vertical step;

The boundary word of a polyomino $P$, denoted by $B d(P)$, is the word obtained by coding its perimeter using the alphabet $A$. For each polyomino, we can have an equivalent class of words that are the conjugates to each other. We choose the representative of these words to be the one that starts from the left lowermost side of the polyomino.

In the last decades, many researchers [3] have studied the digital convexity and worked on finding the link between it and combinatorics on words. In the following two sections, we introduce two families of words, that are essential for this work and we introduce some results.

\subsection{Christoffel words and digital line segments}

The first family of words is Christoffel words. From the geometric point of view, we can consider any Christoffel word as the discretization of a line segment of rational slope $[1,2]$. In this case, we restrict our work to the binary alphabet $A=\{0,1\}$, and start by defining a Christoffel path which is a sequence of unitary steps joining two points of integer lattice. Let $a$ and $b$ be two coprime numbers, the lower Christoffel path of slope $a / b$ is the path joining the origin $O(0,0)$ to the point $(b, a)$ and respecting the following characteristics: 
1. it is the nearest path below the line segment joining these two points;

2. there is no point of $\mathbb{Z}^{2}$ between the path and line segment.

Analogously, the upper Christoffel path is the path that lies above the line segment. By convention, the Christoffel path is exactly the lower Christoffel path. We remark that the upper Christoffel path of slope $a / b$ is the image of the lower Christoffel path obtained by a rotation of angle $180^{\circ}$ (see Figure 1 for example).

Using the binary alphabet $A=\{0,1\}$, and by assigning to each horizontal (resp. vertical) step the letter 0 (resp. 1), we get the Christoffel word $w$ of slope $\frac{a}{b}$, denoted by $C\left(\frac{a}{b}\right)$, where the fraction $\frac{a}{b}$ is exactly $\frac{|w|_{1}}{|w|_{0}}$ (see Figure 2). We define the morphism $\rho: A^{*} \longrightarrow \mathbb{Q} \cup\{\infty\}$ by:

$$
\rho(\epsilon)=1 \text { and } \rho(w)=\frac{|w|_{1}}{|w|_{0}}, \forall w \neq \epsilon \in A^{*} ;
$$

where $\frac{1}{0}=\infty$. This morphism determines the slope for each given word in $A^{*}$. In particular:

1. for $a=0$, we get the Christoffel word of slope $0, C\left(\frac{0}{1}\right)=0$.

2. for $b=0$, we have the Christoffel word of slope $\infty, C\left(\frac{1}{0}\right)=1$.

Christoffel words have several properties and characterizations $[1,8,16]$. Here, we only mention a few of them. Let $\widetilde{w}=w_{n} \cdots w_{2} \cdot w_{1}$ be the reversal of the word $w=w_{1} w_{2} \ldots w_{n}$; we say that $w$ is a palindrome if $\widetilde{w}=w$.

Property 1. The Christoffel word of slope $\frac{a}{b} \neq 0, \infty$ can be written as: $C\left(\frac{a}{b}\right)=$ $0 p 1$, where $p$ is a palindrome.
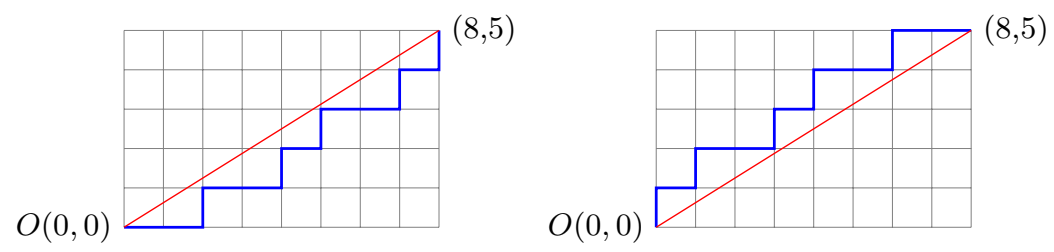

Fig. 1. The lower and upper Christoffel paths of slope 5/8 respectively.

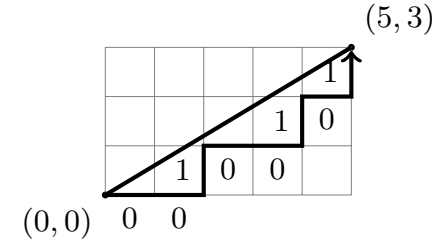

Fig. 2. The Christoffel path from $(0,0)$ to $(5,3)$ and the Christoffel word $C\left(\frac{3}{5}\right)=$ 00100101. 
In addition, any Christoffel word can be factorized into two Christoffel words, using the standard factorization [2] defined as follows.

Property 2. Let $w$ be any Christoffel word of slope $\frac{a}{b}, w$ can be written in a unique way as $w=\left(w_{1}, w_{2}\right)$, where both $w_{1}$ and $w_{2}$ are Christoffel words and of minimal length equal to 1.

Note that the slopes of the new Christoffel words, obtained by the standard factorization, bound the initial slope.

Property 3. Let $w=\left(w_{1}, w_{2}\right)$, such that $\rho\left(w_{1}\right)=\frac{a}{b}$ and $\rho\left(w_{2}\right)=\frac{c}{d}$. We have:

1. $\frac{a}{b}<\rho(w)<\frac{c}{d}$,

2. $\operatorname{det}\left(\rho\left(w_{1}\right), \rho\left(w_{2}\right)\right)=\operatorname{det}\left(\begin{array}{l}\left|w_{1}\right|_{0}\left|w_{2}\right|_{0} \\ \left|w_{1}\right|_{1}\left|w_{2}\right|_{1}\end{array}\right)=c b-a d=1$.

Using this factorization, we are able to define two functions $\phi_{0}$ and $\phi_{1}$ from $A^{*} \times A^{*}$ into itself as follows: $\phi_{0}\left(w_{1}, w_{2}\right)=\left(w_{1}, w_{1} w_{2}\right) ; \phi_{1}\left(w_{1}, w_{2}\right)=\left(w_{1} w_{2}, w_{2}\right)$.

Property 4. Any Christoffel word is constructed by an iteration of these two functions $\phi_{0}$ and $\phi_{1}$ on $(0,1)$.

Consequently, the standard factorization of any Christoffel word is unique, i.e, there exists a unique point of the Christoffel path, where we can do this factorization. This point is exactly the closest point of the Christoffel path with respect to the line segment, that divides the initial Christoffel word into two concatenated Christoffel words. For Figure 2, we note that the standard factorization of $C\left(\frac{3}{5}\right)=(001,00101)$.

\subsection{Lyndon factorization}

The second family of words that is useful for our purpose is the family of Lyndon words [17].

Definition 2. A word $w \in A^{*}$ is a Lyndon word if for all $u, v \in A^{*} \backslash\{\epsilon\}$ such that $w=u . v$, we have $w<v . u$.

In other words, we can say that $w$ is a Lyndon word if $w$ is the smallest word among all its conjugates. Based on this definition, we can deduce one of the properties of this family of words. In fact, Lyndon words are primitive words; otherwise, we can have an equality between at least one of the conjugates of $w$. Therefore, if $w$ is a Lyndon word, $w \cdot w$ is not Lyndon. For example, let $w=0110$, and $X$ be the set of all the possible conjugates of $w$. The smallest element of $X$ in lexicographic order is $w^{\prime}=0011$. Therefore, $w$ is not a Lyndon word. A factorization was introduced in 1958 by Chen, Fox and Lyndon [7].

Definition 3. Every non-empty word $w$ admits a unique factorization as a lexicographically decreasing sequence of Lyndon words: $w=l_{1}^{n_{1}} l_{2}^{n_{2}} \cdots l_{k}^{n_{k}}$, s.t $\rho\left(l_{1}\right)>\rho\left(l_{2}\right)>\cdots>\rho\left(l_{k}\right)$ where $n_{i} \geq 1$ and $l_{i}$ are Lyndon words.

This factorization is called Lyndon factorization. In 1980 Duval proved that this factorization can be computed in a linear time [12]. For example, the Lyndon factorization of $w=100101100101010$ is (1)(001011)(0010101)(0). 


\subsection{Digital convexity by combinatorics}

Now, we introduce the link between these two families of words and digital convexity for polyominos.

Definition 4. A polyomino $P$ is said digitally convex if the convex hull of $P$, $\operatorname{conv}(P)$, satisfies $\operatorname{conv}(P) \cap \mathbb{Z}^{2} \subset P$.

Each digital convex polyomino $P$ can be bounded by a rectangle box. We denote the points of intersection of each side of the bounding box with $P$ by (W)est, (N)orth, (E)ast and (S)outh, starting from the leftmost side and in a clockwise order as shown in Figure 3.

The boundary word of $P, B d(P)$, can be divided into 4 sub-words, $w_{1}, w_{2}, w_{3}$ and $w_{4}$, where each word codes respectively the $W N, N E, E S$ and $S W$ side of $P$. We note that $w_{1}$ starts with the letter 1 , while $w_{2}, w_{3}$ and $w_{4}$ start with 0,3 and 2 respectively. Each word uses only two letters among the four of the alphabet, and hence, we deal on each side with a binary word.

In this section, we consider the WN-word of the boundary word of the digital convex set, and introduce the result of Brleck et al. [3] about digital convexity: a characterization for this path using the two previous families of words.

Property 5. [3] A word $w$ is $W N$-convex if and only if its unique Lyndon factorization $w_{1}^{n_{1}} w_{2}^{n_{2}} \ldots w_{k}^{n_{k}}$ is such that all $w_{i}$ are primitive Christoffel words.

See Fig. 4 for an example of Lyndon factorization: $(1)^{1}(011)^{1}(01)^{2}(0001)^{1}(0)^{1}$ where 1, 011, 01, 0001 and 0 are all Christoffel words. The Christoffel words are arranged in a weakly decreasing order of slopes: $\frac{1}{0}>\frac{2}{1}>\frac{1}{1}>\frac{1}{3}>\frac{0}{1}$. With this result, the WN convex path is decomposed into line segments with decreasing slopes using a linear algorithm [3]. As mentioned before, we know that line segments are represented by Christoffel words, and the decreasing lexicographic order that appears in the Lyndon factorization can explain the condition of decreasing order of slopes.

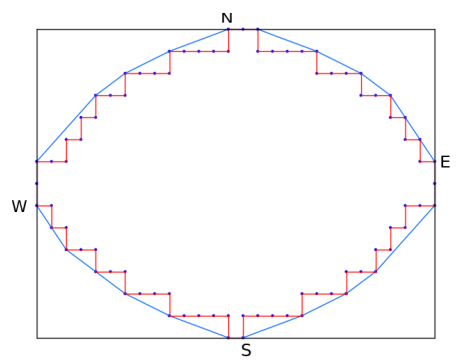

Fig. 3. A digital convex polyomino bounded in a rectangle, where $\mathrm{W}, \mathrm{N}, \mathrm{E}$ and $\mathrm{S}$ are the first points of intersection with the left, upper, right and lower sides of the bounding box. 


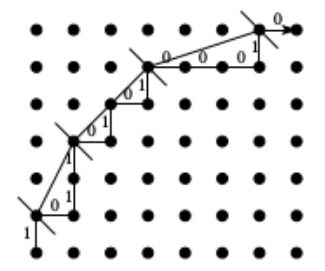

Fig. 4. Example of a $W N$-convex word 101101010001, formed of Christoffel words using the Lyndon factorization.

Due to this, we have the following property:

Property 6. [3] Each vertex of the convex hull of any digital convex set $C$ is exactly at the end of each factor of the Lyndon factorization of its boundary word $B d(C)$.

Such a vertex is exactly at the factor of either 10, 03,32 or 21 , depending on which side of $C$ it is located, either in $W N, N E, E S$ and $S W$ respectively.

\section{Contraction of digitally convex set}

Deflating a digital convex set in a geometric point of view can be obtained by removing, step by step, at most one integer point. In the digital sense, we can see that this deformation is investigated by removing one pixel at each step. In [11], the authors introduced the split operator, that determines the unique accepted position, for each line segment, to add a digital point and conserving the convexity. In this paper, we work on removing digital points under the same condition. Since the vertices, as mentioned before, are digitally represented by the factor 10 in the WN path, then removing a point means switching the factor 10 (resp. 03, 32 and 21) to 01, (resp. 30, 23 and 12).

\subsection{Removable points and contraction}

The points that we are allowed to remove on the boundary are exactly the vertices of the convex hull. In fact, we might face several possibilities depending on the position of the points and the convex shape. In this section, we will mention all the possible situations that we can encounter and the different algorithms that we can use in order to remove a single point from the digital convex set at each step. Given a digitally convex set $C$, on the boundary word $B d(C)$, we can have the vertices of the convex hull of $C$, also called corners. These vertices can be grouped in two categories. The first ones, called the internal vertices, that belong to one of the boundary sub-words. The second ones are external or critical vertices that are at the intersection between two consecutive sub-words. Let $w_{i}$ 
and $w_{i+1}$ be two consecutive sub-words, the corner is exactly the last letter of $w_{i}$ and the first letter of $w_{i+1}$., We define such contraction operator on Christoffel words. Note that Christoffel words on $W N, N E, E S$ and $S W$ sides are binary words over the binary alphabets $\{0,1\},\{0,3\},\{2,3\}$ and $\{1,2\}$ respectively. Christoffel words on each side are defined as follows: $x=0 h 1, y=3 k 0, z=2 l 3$ and $t=1 m 2$ where $h, k, l$ and $m$ are palindroms.

\subsection{Switch operator}

In this section, we consider the four boundary binary sub-words $w_{1}, w_{2}, w_{3}$ and $w_{4}$ that belong to $A^{*}=\{0,1,2,3\}^{*}$. On these sub-words, we set an operation at a certain position $k$, from $A^{*}$ into $A^{*}$ such that it switches the two consecutive letters of this word at position $k$.

Definition 5. Let $w=l_{1} l_{2} \ldots l_{k} l_{k+1} \ldots l_{n}$ be a word in $\{0,1,2,3\}^{*}$,

$$
\operatorname{switch}_{k}(w)=l_{1} l_{2} \ldots l_{k+1} l_{k} \ldots l_{n}
$$

where $02=20=13=31=\epsilon$.

Given a boundary word $B d(C)$, thanks to Property 6, we can apply this switch operator to $B d(C)$ at the last letter of each Christoffel word obtained by the Lyndon factorization of $B d(C)$. In this way, we remove a corner of the form 10, 03, 32 or 21 . We have two cases, depending on the position of a corner. The first case is when the corner belongs to one of the four paths, either $W N, N E, E S$ or $S W$. In this case, the operation is directly applied at position $k$ of the Christoffel word $w$, where $k=|w|$, as seen in Fig. 5. In the second case, we deal with the corners that are at the intersection position between $w_{1}, w_{2}, w_{3}$ and $w_{4}$. In fact, only at these positions, it might occurs some particular situations. We might obtain, after a switch operation, one of the factors $02,20,13$, and 31 that will be replaced by $\epsilon$ by definition. But in this case, a re-arrangement for the four boundary words has to be considered; see an example in Fig. 6 .

To keep the set 4-connected, we must avoid the existence of the factor $1^{k} 0^{l} 3^{k} 2^{l}$ and its conjugates on the boundary word. Therefore, if after a certain
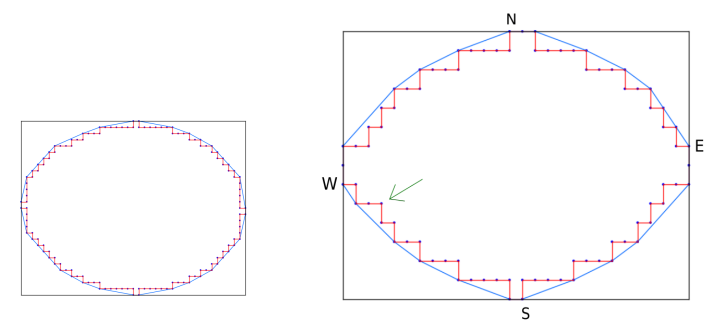

Fig. 5. The fourth boundary word of $C$ is: $w_{4}=(2)(12222)(1222)(122)^{2}(12)^{2}$. The switch operator is applied on the fifth factor, at position 2 . We obtain the new boundary word $w_{4}^{\prime}=(2)(12222)(1222)(122)(12122)(12)$. 

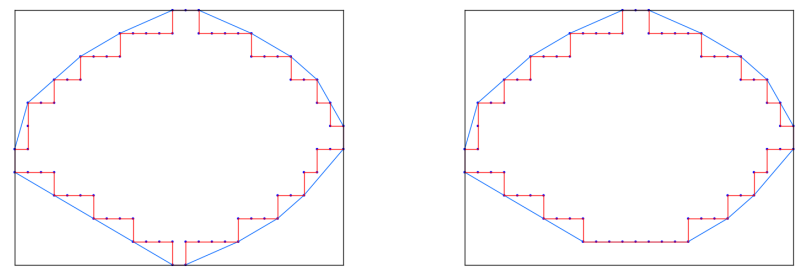

Fig. 6. Re-arrangement of the boundary words, $w_{4}$ and $w_{3}$, after removing the factor 31 from the boundary word $B d(C)$ of the digital convex set $C$.

operation, one of these four factors appear in the boundary word, the switch operation must be canceled.

By applying the switch operator locally all around the boundary word and sub-words, the possible words that can be obtained are of the following form:

Theorem 1. Let $u$ and $v$ be two consecutive Christoffel words such that $|u|=$ $k$, and $\rho(u)>\rho(v)$. By applying the switch operator one of these cases appears:

1. $\operatorname{switch}_{k}(u v)=w^{\ell}$ where $\rho(w)<\rho(u)$ and $\ell \geq 1$;

2. $\operatorname{switch}_{k}(u v)=m_{1}^{\prime} \ldots m_{i}^{\prime}$ where $\rho\left(m_{1}^{\prime}\right)<\rho(u)$ and $\rho\left(m_{1}^{\prime}\right)>\ldots \rho\left(m_{i}^{\prime}\right)$.

In order to give the proof, we must recall Pick's theorem [19] that gives the area of a triangle using its interior and boundary integer points:

Theorem 2. [18] The area of a given polygon in Euclidean plane, whose vertices have integer coordinates, is equal to $A=i+\frac{b}{2}-1$ where $i$ is the number of interior integer points and $b$ is the number of boundary integer points of the polygon.

After this theorem, we can pass to a sketch of the proof of Theorem 1.

Proof. From Properties 2 and 3, we write $m_{1}=u . v$ and $m_{2}=x . y$ with $\rho(u)<$ $\rho\left(m_{1}\right)<\rho(v)$ and $\rho(x)<\rho\left(m_{2}\right)<\rho(y)$. Recall that the area of a triangle $A B C$ is equal to $\frac{1}{2} \operatorname{det}(\overrightarrow{A B}, \overrightarrow{A C})=\frac{1}{2} \operatorname{det}\left(\begin{array}{l}x_{\overrightarrow{A B}} x_{\overrightarrow{A C}} \\ y_{\overrightarrow{A B}} y_{\overrightarrow{A C}}\end{array}\right)=i+\frac{b}{2}-1$. Hence, we can conclude that: $i=\frac{\operatorname{det}(\overrightarrow{A B}, \overrightarrow{A C})-b+2}{2}$. With a simple vectorial calculation we can conclude that if $\operatorname{det}\left(\rho\left(m_{1}\right), \rho\left(m_{2}\right)\right)=1$, we get $i=0$. Hence we get the first case for $\ell=1$ since in this case we get $b=3$, that are exactly the points $A, B$ and $C$. We can note that this result is the inverse of the split operator defined in [11]. Similarly, and by noticing that each Christoffel word is obtained by applying several morphisms of $\phi_{0}$ and $\phi_{1}$ defined in Property 4 , we can prove the other cases.

Some examples are illustrated in Fig. 7.

The number of possible new segments is related to the solutions of the a variant of the Knapsack problem in integer linear programming: given a triangle 

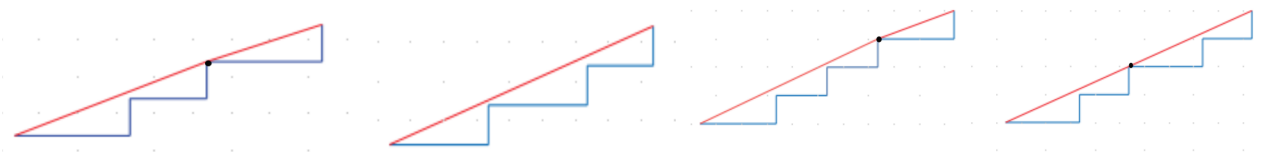

$\operatorname{switch}_{6}\left(C\left(\frac{2}{5}\right) \cdot C\left(\frac{1}{3}\right)\right)=C\left(\frac{3}{8}\right)$.

$\operatorname{switch}_{9}\left(C\left(\frac{2}{7}\right) \cdot C\left(\frac{1}{3}\right)\right)=\left(C\left(\frac{2}{5}\right)\right)^{2}$.

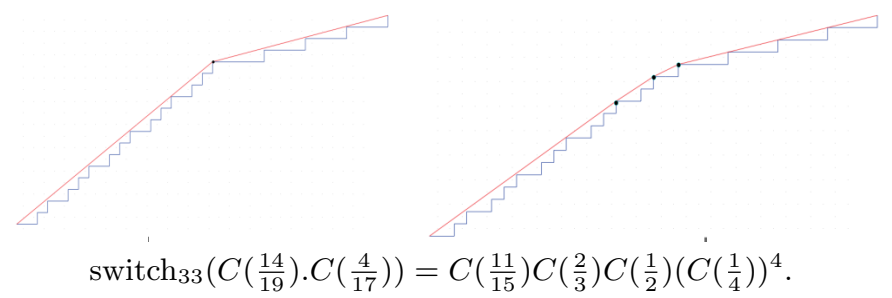

Fig. 7. Examples of three different cases of switch operator.

$T$ with rational vertices in a $[0, N] \times[0, N]$ domain $(N \in \mathbb{Z})$, the number of vertices in the convex hull of $T \cap \mathbb{Z}^{2}$ is in $O(\log N)$ [14]. This result has been used to efficiently compute the convex hull of interior grid points of rational convex polygon [6]. In other words, the number of $m_{i}^{\prime} s$ and the value of $k$ is bounded by $O(\log N)$.

\section{Choosing the removal order}

As mentioned above, we can find all points that can be removed in order to deflate a digital convex set $C$ while preserving its convexity. These points are exactly the vertices of the convex hull of $C$. Here arises the natural question: is there any specific order for choosing which digital point to remove at each step? If so, is there a preferred order? In fact, we investigate and test three ordering techniques to deflate $C$.

Let $V$ be a removable point, and $V_{p}$ and $V_{n}$ be its previous and next vertices of the convex hull of $C$. After removing $V$ from $C$, let us assume that the set of new vertices $\mathbf{W}$ is obtained, instead of $V$.

\subsection{Ordering by determinant obtained from two consecutive line segments}

For each removable point $V$, we calculate

$$
\triangle(V)=\frac{1}{2} \operatorname{det}\left(\overrightarrow{V_{p} V}, \overrightarrow{V V_{n}}\right),
$$

which is the (signed) area of triangle $V_{p} V V_{n}$. Then, all removable points are listed in increasing order with respect to this area measure, and the point with min- 
imum value is chosen for each elementary contraction step. The list is updated locally whenever a point is removed.

\subsection{Ordering by area change}

For each removable point $V$, we calculate

$$
\triangle_{c}(V)=\triangle(V)-\frac{1}{2} \sum_{W \in \mathbf{W}} \operatorname{det}\left(\overrightarrow{V_{p} W}, \overrightarrow{W W_{n}}\right)
$$

which is the area difference between the convex hulls of $C$ and $C \backslash\{V\}$. Similarly to the previous case, all removable points are listed in increasing order with respect to this area change, and the point with minimum value is chosen for each elementary contraction step. Once a point is removed, the list is updated locally.

\subsection{Ordering by angle-sum change}

For each removable point $V$, we calculate the difference between the line-segment angle at $V$ and the sum of such angles at all $W \in \mathbf{W}$, such that

$$
\theta_{c}(V)=\theta(V)-\sum_{W \in \mathbf{W}} \theta(W)
$$

where

$$
\theta(V)=\cos ^{-1}\left(\frac{\overrightarrow{V_{p} V} \cdot \overrightarrow{V V_{n}}}{\left\|\overrightarrow{V_{p} V}\right\|\left\|\overrightarrow{V V_{n}}\right\|}\right) .
$$

Similarly to the previous cases, all removable points are listed in increasing order with respect to this angle measure, and the point with minimum value is chosen from the list for each elementary contraction step. Once a point is removed, the list is updated locally.

\section{Experimental results}

Deforming digitally convex sets with digital convexity preservation is crucial, and has different applications in digital image processing. One of them is rotating digitally convex sets without loss of their convexity, which is not trivial at all.

Figure 8 shows the experimental results of applying our methods to a rotated digitized ellipse, which was originally digitally convex before the rotation but is not anymore (see Fig. 8 (b)). In order to repair the digital convexity, we made the convex hull and digitized it (see Fig. 8 (c)). As the difference between the rotated digitized ellipse and the digitized convex hull are 45 pixels, which are colored in gray in Fig. 8 (b), we decided to remove 45 pixels while preserving the digital convexity by using our method with the three different strategies. Note that, in Section 4, the point to be removed at each iteration has to be with 


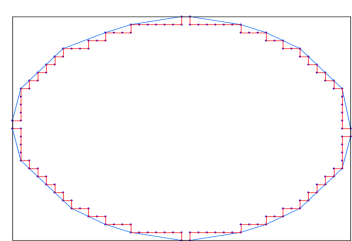

(a)

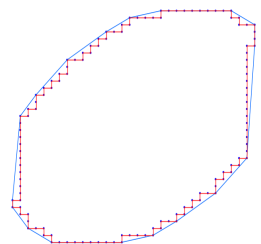

(d)

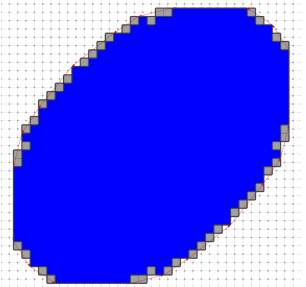

(b)

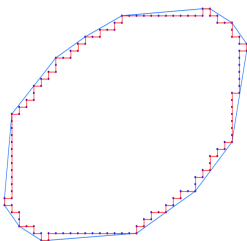

(e)

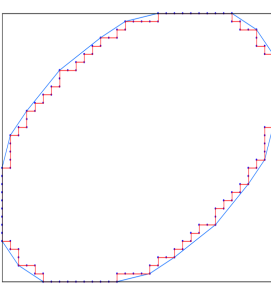

(c)

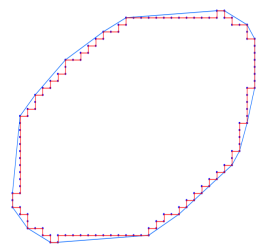

(f)

Fig. 8. (a) A digitized ellipse, which is digitally convex; (b) its rotated image with angle $\frac{\pi}{4}$ (in blue), which is not anymore digitally convex; (c) the convex hull of the rotated shape of (b) where the difference from the rotated image is visualized with 45 gray pixels in (b); the contraction results for the digitized convex hull of the rotated digitized ellipse of (b) after removing 45 points with the three ordering strategies: by (d) determinant, (e) area change, and (f) angle-sum change.

a minimal value for the three strategies. We might encounter the case where several points can give us the same minimal value. Our algorithm chooses in a random way a removal point among all the points having the minimal value at each iteration. Using this algorithm, the results after 45 iterations can be seen in Fig. 8 (d,e,f), respectively. Table 1 also shows the results of the three different strategies after 22, 150 and 200 iterations.

While all of them preserve digital convexity, the deformation tendencies are slightly different. The first removal-point ordering strategy based on area tends to extend vertical and horizontal line segments while it keeps curved parts as well. On the other hand, it is seen that the second (resp. third) strategy based on area (resp. angle) change tend to straighten curved parts.

\section{Conclusion}

We presented the method for contraction of digitally convex sets by removing digital points one by one while preserving their digital convexity, with a help of combinatorics on words. Such tools enable us to detect a set of removable points and to define such convexity-preserving contraction of a digital set as an operation of re-writing its boundary word. In order to choose one of removable 
Table 1. The contraction results for the digitized convex shape illustrated in Fig. 8 (c) after removing 22, 150 and 200 points with the three ordering strategies: determinant, area change, and angle-sum change.

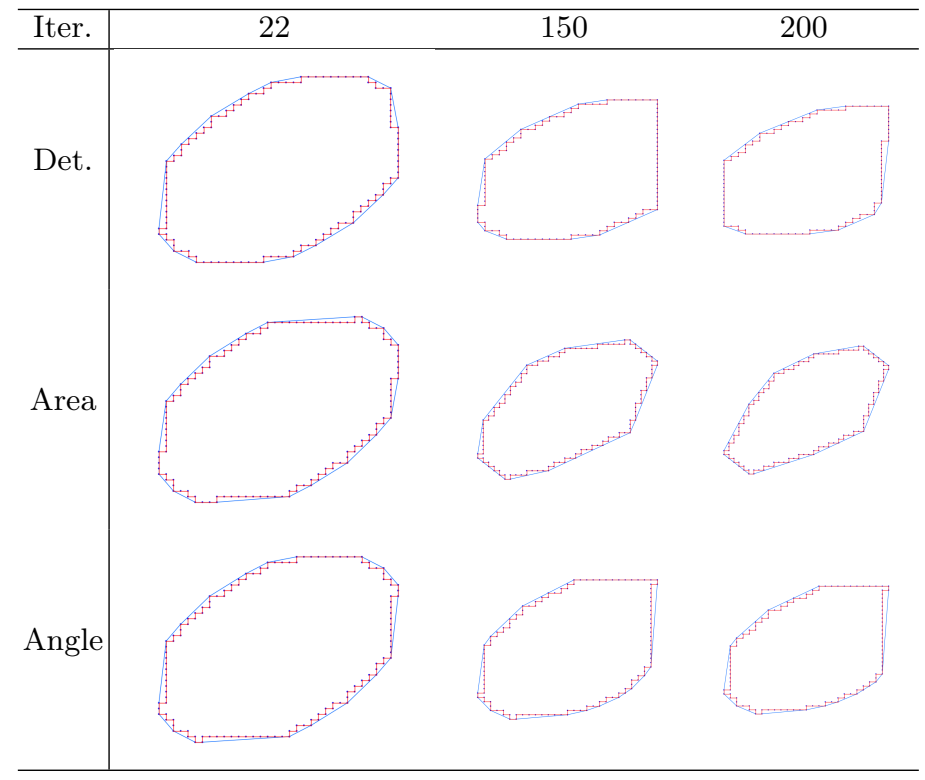

points for each contraction step, we present three geometrical strategies, which are related to vertex angle and area changes of the convex hull of a digitally convex set. We showed experimental results of applying the methods to repair some non-convex digital sets, which are obtained by a rotation of convex digital sets. Our further work will consist of deforming non-convex sets while preserving their convex and concave parts. Another idea can be to write the algorithm needed to add a point at each step instead of removing.

\section{References}

1. J. Berstel, A. Lauve, C. Reutenauer, and F. Saliola. Combinatorics on words: Christoffel words and repetition in words. 2008.

2. J-P. Borel and F. Laubie. Quelques mots sur la droite projective réelle. Journal de théorie des nombres de Bordeaux, 5(1):23-51, 1993.

3. S. Brlek, J-O. Lachaud, X. Provençal, and C. Reutenauer. Lyndon + christoffel=digitally convex. Pattern Recognition, pages 42-10, 2009.

4. G. Castiglione, A. Frosini, E. Munarini, A. Restivo, and S. Rinaldi. Combinatorial aspects of l-convex polyominoes. European Journal of Combinatorics, 28(6):17241741, 2007.

5. G. Castiglione, A. Frosini, A. Restivo, and S. Rinaldi. Enumeration of l-convex polyominoes by rows and columns. Theoretical Computer Science, 347(1-2):336$352,2005$. 
6. E. Charrier and L. Buzer. Approximating a real number by a rational number with a limited denominator: A geometric approach. Discrete Applied Mathematics, 157(16):3473-3484, 2009 .

7. K. Chen, R. Fox, and R. Lyndon. Free differential calculus, iv. the quotient groups of the lower central series. Annals of Mathematics, 68:81-95, 1958.

8. E. Christoffel. Observatio arithmetica. Annali di Matematica Pura ed Applicata (1867-1897), 6(1):148-152, 1875

9. A. Del Lungo, E. Duchi, A. Frosini, and S. Rinaldi. Enumeration of convex polyominoes using the eco method. In DMCS, pages 103-116, 2003.

10. A. Del Lungo, E. Duchi, A. Frosini, and S. Rinaldi. On the generation and enumeration of some classes of convex polyominoes. the electronic journal of combinatorics, 11(1):R60, 2004.

11. P. Dulio, A. Frosini, S. Rinaldi, L. Tarsissi, and L. Vuillon. First steps in the algorithmic reconstruction of digital convex sets. In International Conference on Combinatorics on Words, pages 164-176. Springer, 2017.

12. J. Duval. Mots de lyndon et périodicité. RAIRO, Informatique théorique, 14(2):181-191, 1980.

13. H. Freeman. On the encoding of arbitrary geometric configurations. IRE Transactions on Electronic Computers, (2):260-268, 1961.

14. A. C. Hayes and D. G. Larman. The vertices of the knapsack polytope. Discrete Applied Mathematics, 6(2):135-138, 1983.

15. R. Klette and A. Rosenfeld. Digital Geometry: Geometric Methods for Digital Picture Analysis. Morgan Kaufmann Publishers Inc., San Francisco, CA, USA, 2004.

16. M. Lothaire. Algebraic combinatorics on words, volume 90 of Encyclopedia of Mathematics and its Applications. Cambridge University Press, Cambridge, 2002.

17. R. Lyndon. Identities in finite algebras. Proceedings of the American Mathematical Society, 5(1):8-9, 1954.

18. M. Minsky and S. Papert. Perceptrons. MIT Press, Cambridge, MA, 1969.

19. G. Pick. Geometrisches zur zahlenlehre. Sitzungsberichte des Deutschen Naturwissenschaftlich-Medicinischen Vereines für Böhmen "Lotos" in Prag., v.4748 1899-1900, 1906. 